\title{
Publisher Correction: How fruit ripening is ENCODEd
}

Federico Scossa and Alisdair R. Fernie

Correction to: Nature Plants https://doi.org/10.1038/s41477-018-0272-0, published online 24 September 2018.

In the version of this News \& Views originally published, the ripening mode of Prunus persica was incorrectly listed as 'MADS-type loop' in Fig. 1, the correct ripening mode is 'NAC-type loop'. In addition, the authors would like to remove 'H3K4me' as a main epigenetic mark in ripening genes for Musa acuminata, Solanum lycopersicum and Prunus persica in Fig. 1, as this marker is common in all species. This figure and caption have now been amended in all versions of the News \& Views.

Published online: 18 October 2018

https://doi.org/10.1038/s41477-018-0303-x 